\title{
EXPERIMENTAL STUDY OF THE EFFECT OF THE WAKE GENERATED BY OSCILLATING GURNEY FLAP
}

\author{
J. MARAÑON DI LEO ${ }^{\dagger \dagger}$, M.A. MARTINEZ, J. S. DELNERO ${ }^{\dagger}$ and M. O. GARCIA SAÍNZ \\ $\uparrow$ UIDET Capa Límite y Fluidodinámica Ambiental (LaCLyFA), Facultad de Ingeniería, UNLP. Calle 116 e/47 y 48 \\ - (1900) La Plata - Pcia. de Bs. As. - Argentina. \\ $\ddagger$ Consejo Nacional de Investigaciones Científicas y Técnicas, Avda. Rivadavia 1917, CP C1033AAJ, Cdad. de Bue- \\ nos Aires, Argentina. \\ jmaranon@ing.unlp.edu.ar
}

\begin{abstract}
The study of the turbulent near wake of an airfoil provided with flow control devices shows the flow configuration in such conditions and the starting vortices mechanism, while, the far wake offers information about the general performance of the model. The objective of the present work is to study the develop of the fluid-dynamic structures found in the NACA 4412 airfoil wake, as well as the development of the same structures when flow control techniques are applied by means of a oscillating Gurney Flap place in the lower surface of the wing model, close to the trailing edge. Tests were performed at a given Reynolds numbers and the flow control system was set at different frequencies. In order to study the effect of the control mechanism on the wake, hot wire anemometry techniques were used. Two components of the velocity vector were measured - longitudinal and vertical - by means of a vertical array of three sensors acquiring simultaneously. The intention is to quantify the flow general configuration at the airfoil wake for different test conditions. The velocity vectors will be analyzed, together with the turbulence intensity and integral scales.
\end{abstract}

Keywords-- Wind tunnel, Aerodynamic, Detachments, Gurney flap, Hot-wire anemometry.

\section{INTRODUCTION}

The usage of high-lift devices to increase the lift capacity of wings has been one of the main goals of re-search in the field of flying, since the beginnings of aviation. Almost all the airplanes use some kind of high-lift system (also cars, motorboats and wind generators).

The main challenge in this field is to achieve a significant increase in lift without a significant increase in drag. Modern technologies allow to implement passive and active control systems which promise to improve their efficiency, exceeding the classical ones.

One of those high-lift devices is the so-called Gurney Flap (Jeffrey and Hurst, 1996). Several research works demonstrated that the near wake after an airfoil provided with a mini-flap Gurney resembles an asymmetric vortex street with vortex structures of different sizes producing fluctuations in the global airfoil circulation (Mahrt, 1991). Focusing on the wake of an airfoil, it is clear that an increase in lift is associated to an increased downwash. This re-quires a higher asymmetry of the flow in the near wake.
In the near wake of a wing airfoil provided with Gurney mini-flaps, in lift conditions, the starting vortices detached from the upper surface must show a different intensity than those detached from the lower surface. This asymmetry assures the mean deviation of the flow associated the lift increment provoked by those mini-flaps (Giguerè et al., 1995; Casper et al., 2012). The existence of the counter-rotating asymmetric vortices, in the near wake of an airfoil provided with trailing edge Gurney mini-flaps, was experimentally demonstrated. (Boldes $e t$ $a l ., 2010)$ In an experimental and numerical study of the near wake of a lifting airfoil, (Hah and Lakshminarayana, 1982), confirmed this behavior as they found that the wake becomes symmetric just one chord-length downstream the trailing edge.

One of the aspects that must be considered, when analyzing the effect of this sort of device, are the characteristics of the incoming flow, such as turbulence intensity, space scales and time scales, for the same mean velocity. Experimental results were obtained about the behavior of Gurney flaps placed at the trailing edge of an airfoil, about the effect of different turbulent flows on the lift and drag coefficients, compared with a quasi-laminar flow. Experiments also clarified the effect of turbulence on Gurney mini-flaps of different sizes placed at several distances from the trailing edge (Colman et al., 2010). All these results were obtained analyzing the wake produced by the airfoil with GMF. Similar results were found in numerical simulations (Wassen et al., 2008).

The question arising now is how implement flow control techniques to eliminate, or to weaken, the vortices that produce negative circulation, and to strengthen the others, in order to increase circulation and lift. This fact would result in an increase in the mean flow deviation and in the downwash in the near wake.

Fluid-dynamical models approximate the effect of the oscillating flap to the superposition of the effects of a curved airfoil with the effects of the displacement of the rear stagnation point, generating a Von Kármán wake. However, these models do not consider the difference between the near wake and the far wake. A more accurate analysis shows that the previously mentioned effect is found in the near wake, where two lines of counter-rotating vortices with asymmetric strength are shed. Beyond one chord length the wake is stabilized resembling a classical Von Kármán symmetric vortex shedding.

In order to attain a drag reduction by "stabilizing the 
wake" some authors suggest the use of span-wise holes, slits, serrated flaps and wake-bodies (Van Dam et al., 1999), while other recommend to eliminate straightforward the double row of counter-rotating vortices of the wake be-hind a Gurney type miniflap (Schatz et al., 2004).

Two shear layers emerge alternatively from the root and from the tip of a GMF rolling up into a pattern of counter rotating vortices, establishing an absolute wake instability. When an absolutely unstable scenario exists, any arbitrary disturbance injected into the flow propagates upstream and/or downstream. Therefore, it is expected that the vortex structures generated behind the airfoil are able to influence the upstream and downstream conditions.

It is quite evident that, for an airfoil provided with GMF, the vortex line shed behind the trailing edge must be also connected to the overall circulation around the airfoil. The strength of the vortices generated by the rolling up of the lower surface shear layer is connected to an augmented circulation of the bound vortex, promoting a lift increase. This cannot be counteracted by the lift decrease due to the weaker opposite circulation connected to the counter rotating rolling up shear layer detached from the upper surface.

For all these reasons we propose to study the far wake and the near wake of an airfoil provided with GMF, immersed in turbulent flow. Different configurations of the model will be tested, in order to find the flow asymmetries and to explain the previously mentioned behavior and the influence of turbulence. It is important to note that there are few works studying the aerodynamic behavior and the wake structure of airfoils provided with active and/or passive flow control devices, such as GMF, immersed in free turbulent flow (Gad-el-Hak, 2001). The shear layers related to active GMF, in the wake of the airfoils, depend significantly on the characteristics of the upstream free flow profile, as well as on the device oscillation frequencies. This implies the need for a more detailed measuring of the fluid dynamics wake structure, until a minimum of one chord length behind the airfoil. This is the motivation of this work.

\section{METHODS}

\section{A. General}

In order to achieve the previously defined objective, the effect on its wake of an oscillating flap placed in an aerodynamic airfoil, will be studied. A NACA 4412 air-foil provided with a GMF driven by an electro-mechanic system was chosen. The GMF was placed near the trailing edge of the airfoil on the pressure surface. Instant velocities in three different points will be simultaneously measured with a hot wire anemometer, in order to quantify the effect of the flap on the wake. Instant and mean velocities, turbulence intensity, spatial and time scales and frequency spectra will be analyzed.

Temporal and spatial integral scales were calculated from the autocorrelation coefficients analysis (Eq. 2). The autocorrelation function $(R u)$ (Eq. 1) and its coefficient $C(t)$ (Eq. 2) are defined as follows:

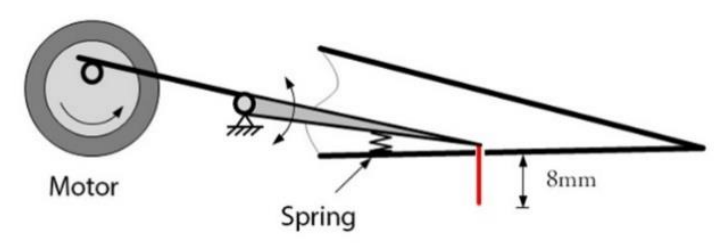

Figure 1. Electro-mechanic GMF actuation system.

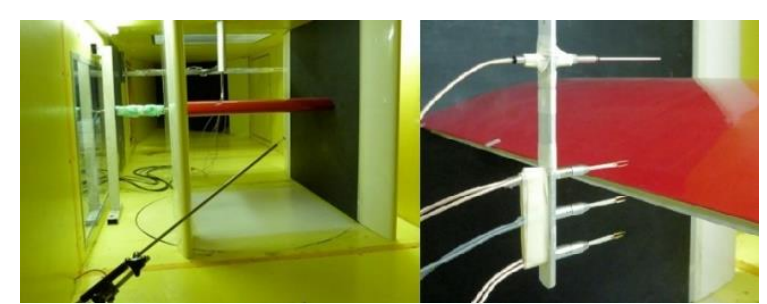

Figure 2. Model and hot wire anemometer probes.

$$
\begin{gathered}
R_{u^{\prime}}(\Delta t)=\lim _{T \rightarrow \infty} \frac{1}{T} \int_{0}^{T} u^{\prime}(t) \cdot u^{\prime}(t+\Delta t) \cdot d t \\
C(\Delta t)=\frac{R_{u^{\prime}}(\Delta t)}{\sigma_{u^{\prime}}^{2}}
\end{gathered}
$$

where $\sigma_{u^{\prime}}^{2}$ is the variance and is defined as:

$$
\sigma_{u^{\prime}}^{2}=\lim _{T \rightarrow \infty} \frac{1}{T} \int_{0}^{T} u^{\prime 2}(t) \cdot d t
$$

The autocorrelation coefficient function takes the unity value at time $t=0$, when the acquisition starts, and trends to zero when the interval of time goes to infinity.

The time gap between $t=0$, and the time when the autocorrelation coefficient $C(t)$ takes the value $1 / e$, for the first time, give an approximated value of the integral time scale $\left(\lambda_{t}\right)$. With this scale, and the mean value of the flow component under analysis, the spatial integral turbulent scale $(\lambda)$ can be calculated, using the Eq. 4 .

$$
\lambda=\bar{V} \cdot \lambda_{y}
$$

Additionally, the turbulence intensity $(T i)$ was calculated as follows:

$$
T i_{u}=\frac{\sigma_{u^{\prime}}}{V} ; T i_{v}=\frac{\sigma_{v^{\prime}}}{V}
$$

\section{B. Facilities and Test Conditions}

The tests were conducted in the wind tunnel of the UIDET-LaCLyFA at the National University of La Plata (Delnero et al., 2005). It is a closed circuit wind tunnel, with a test section of $1.4 \mathrm{~m} \mathrm{x} 1.0 \mathrm{~m} \mathrm{x} 7.5 \mathrm{~m}$ and $V_{\max }=$ $20 \mathrm{~m} / \mathrm{s}$.

The model was based on a NACA 4412 airfoil and was built with a wooden skeleton, with aluminum inserts, all covered with a high impact polystyrene sheet. This kind of structure gave enough interior space to lodge the electro-mechanic crank-connecting rod system, driving the aluminum made gurney flap. The engine r.p.m. can be varied and so the GMF oscillation frequency, up to a maximum of $30 \mathrm{~Hz}$. The chord length $(\mathrm{C})$ of the model is $400 \mathrm{~mm}$ and its span $800 \mathrm{~mm}$. The GMF is placed at $10 \% \mathrm{C}$ from the trailing edge and its length is $1.5 \% \mathrm{C}$, as shown in Fig. 1.

The model was mounted in the wind tunnel between two panels to ensure the flow bi-dimensionality. A scheme is shown in Fig. 2. The measurement of the instantaneous velocity field was performed using a hot wire anemometry system (CTA Dantec Streamline), with a 


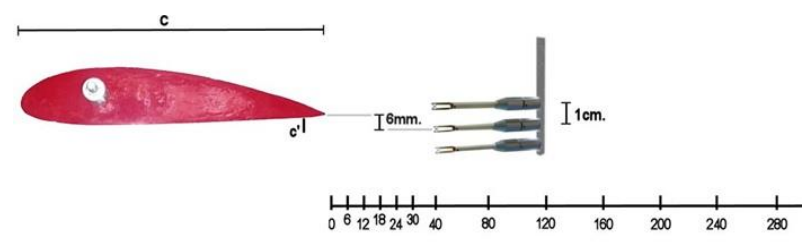

Figure 3. Measurement points (upper, middle and lower row positions).
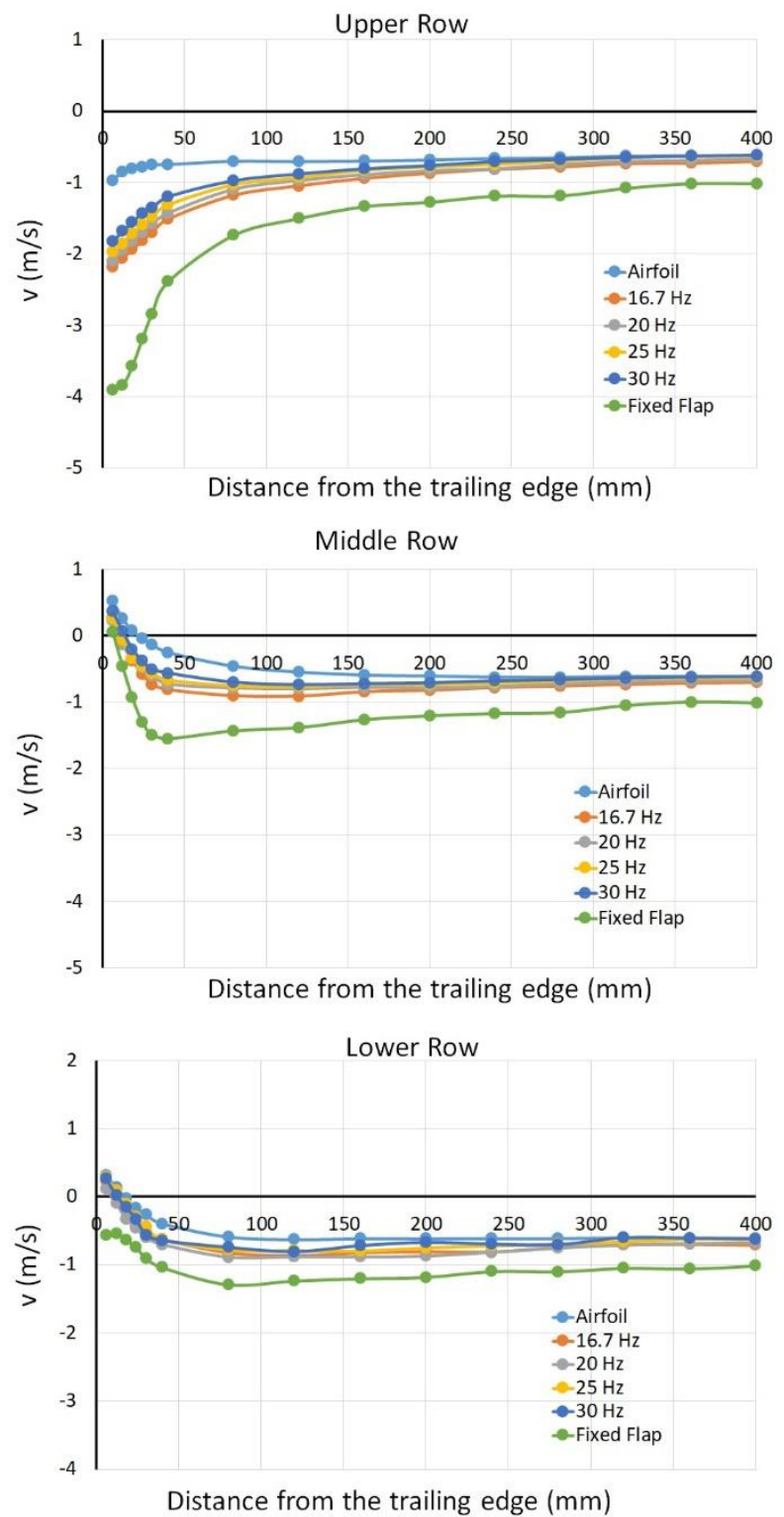

Figure 4. $v$ mean velocity distribution (upper-middle-lower) row- $\alpha=0^{\circ}$.

dual sensor (fiber film probes 55R51). The acquisition was made at a sample frequency of $2 \mathrm{kHz}$, using a lowpass band filter at $1 \mathrm{kHz}$, acquiring 16,384 samples in each test. The vertical $(V)$ and longitudinal $(u)$ components of velocity were simultaneously measured at three points (upper, middle and lower row positions), by means of and arrange of three probes. The probes were placed at a vertical line, separated $10 \mathrm{~mm}$ from one another (Fig. 2).
Tests were performed at a free stream velocity of $10 \mathrm{~m} / \mathrm{s}$. Two angles of attack were used, $0^{\circ}$ and $2^{\circ}$. Six different configurations were tested: plain model (no GMF), fixed GMF and oscillating GMF at four different frequencies $(16,20,25$ and $30 \mathrm{~Hz})$. The incoming flow was found to be turbulent, and $2 \%$ turbulence intensity was calculated in the surroundings of the model.

\section{RESULTS}

\section{A. Velocity analysis}

Results from the processing and analysis of the acquired data are shown by means of mean velocities, scales, intensities and frequency spectra for the two velocity components. Mean velocity distribution for the six configurations are plotted along the wake. Both components $(u$ and $v$ ) are shown in the following figures.
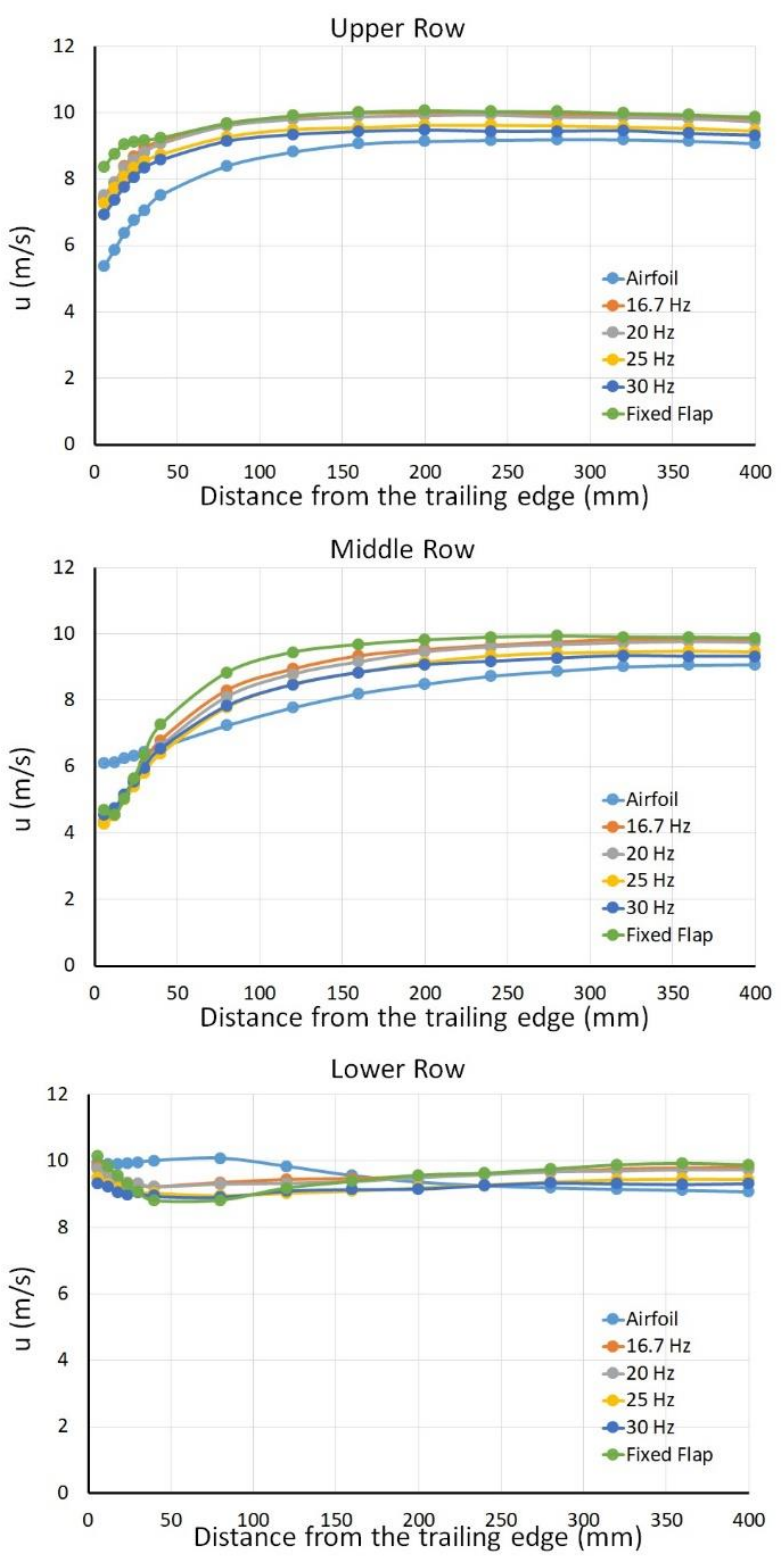

Figure 5. $u$ mean velocity distribution (upper-middle-lower) row- $\alpha=0^{\circ}$. 

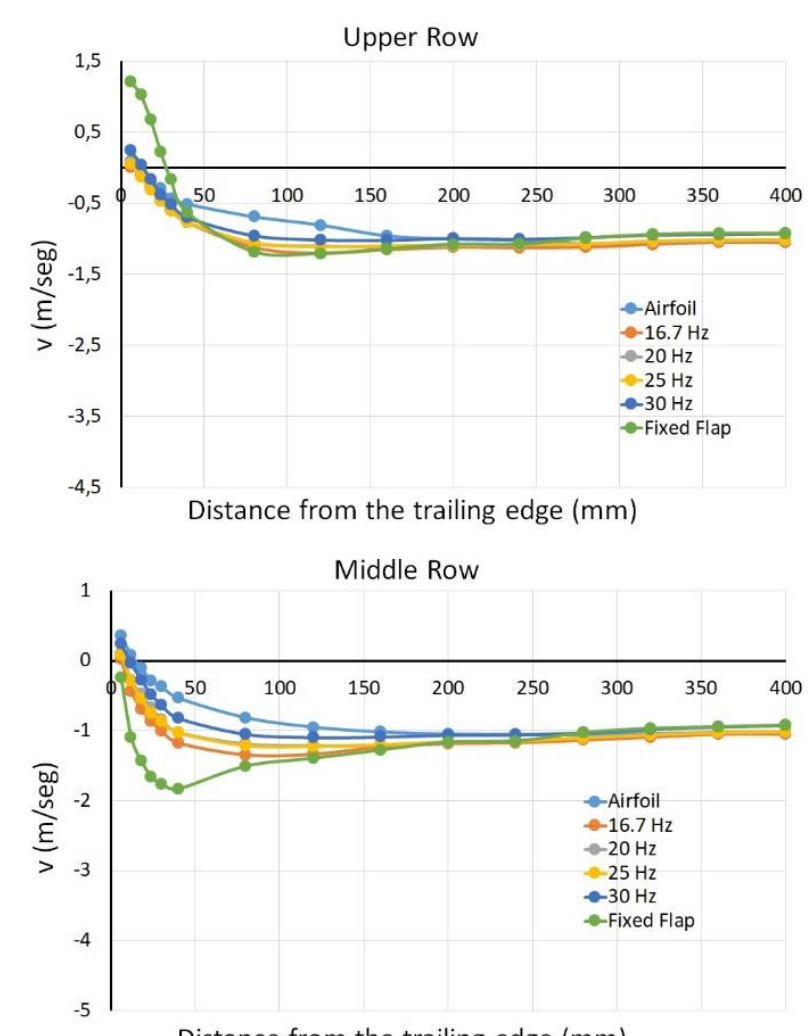

Distance from the trailing edge $(\mathrm{mm})$

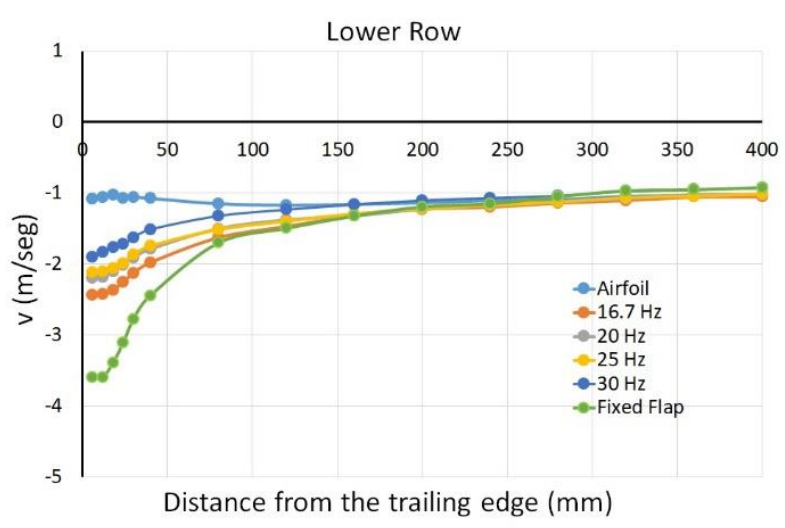

Figure 6. $v$ mean velocity distribution (upper-middle-lower) row $\alpha=2^{\circ}$.

The $u$-component is seen to increase in all configurations as measured farther from the trailing edge. However, the greatest changes are observed in the $\mathrm{V}$-component, showing different behaviors in the plain airfoil, in the fixed GMF and in the oscillating GMF. The fixed GMF generates the greatest vertical velocities, thus, the greatest downwash, being responsible for the lift and drag increases.

The oscillating GMF generates higher downwash in the far wake, while, the plain airfoil generates downwash in the near wake. The behavior did not show remarkable differences related to the oscillation frequencies. According to the (Eq. 5), the v component velocity turbulence intensity distributions are calculated for the near and far fields at the six different test conditions. Results are plotted in Figures 6-10.
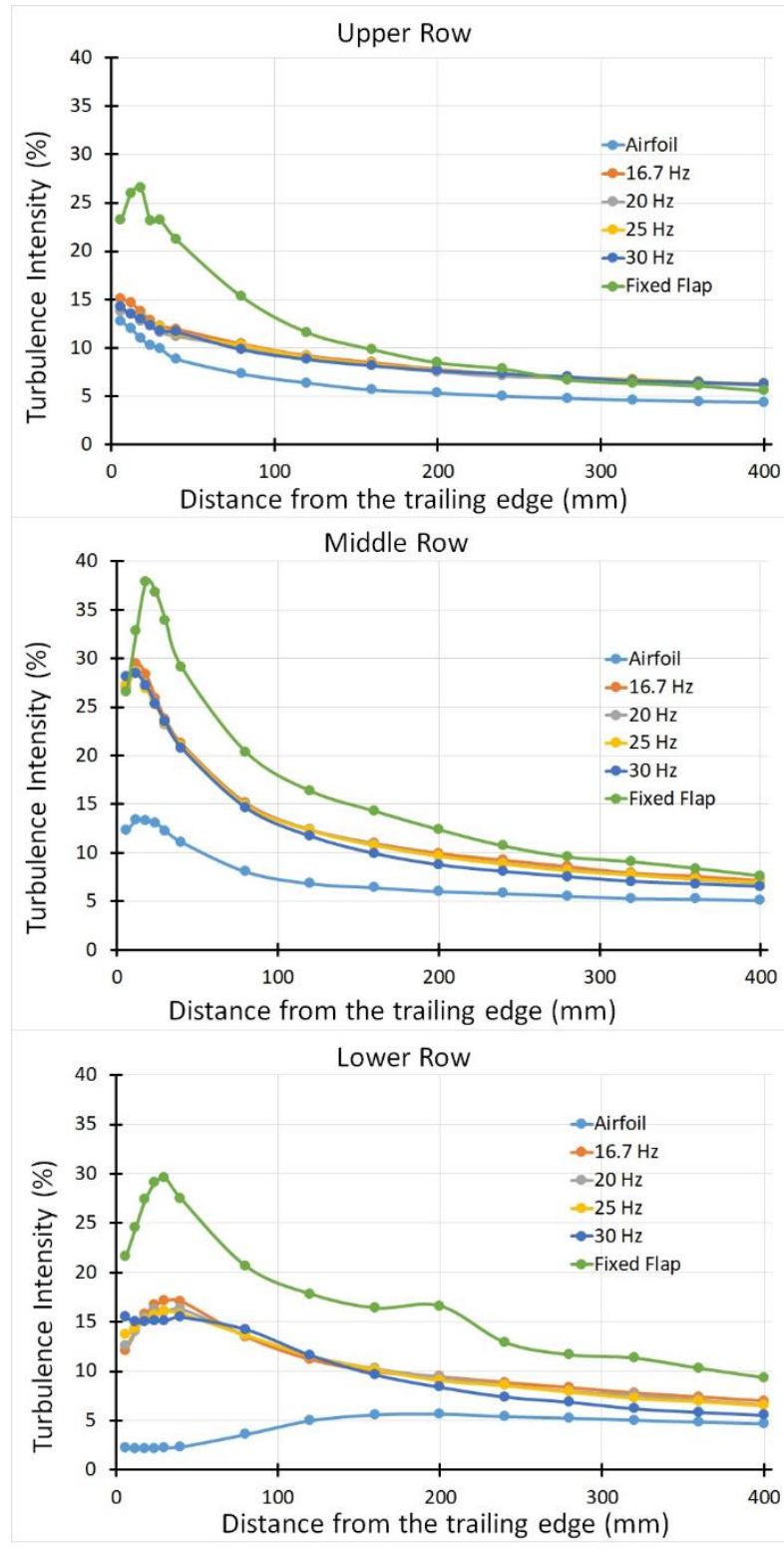

Figure 7. Turbulence Intensity $-\alpha=0^{\circ}$.

In all cases the turbulence intensity values diminish and converge to a unique value in the far wake. In the configurations with oscillating GMF the slope is more pronounced that in the fixed device. The wake produced by the oscillating configurations is less turbulent than the one produced by the fixed flap. Again, no remarkable differences are shown due to the oscillation frequency. When the angle of attack is increased a slight decrease is observed in the near wake. In the far wake the behavior remains unaltered and constant.

\section{A. Autocorrelation Analysis}

The spatial integral turbulent scales were determined from autocorrelations results and applying frozen flow theory (Delnero et al., 2005). The distribution of the autocorrelation function at null angle of attack is shown for 

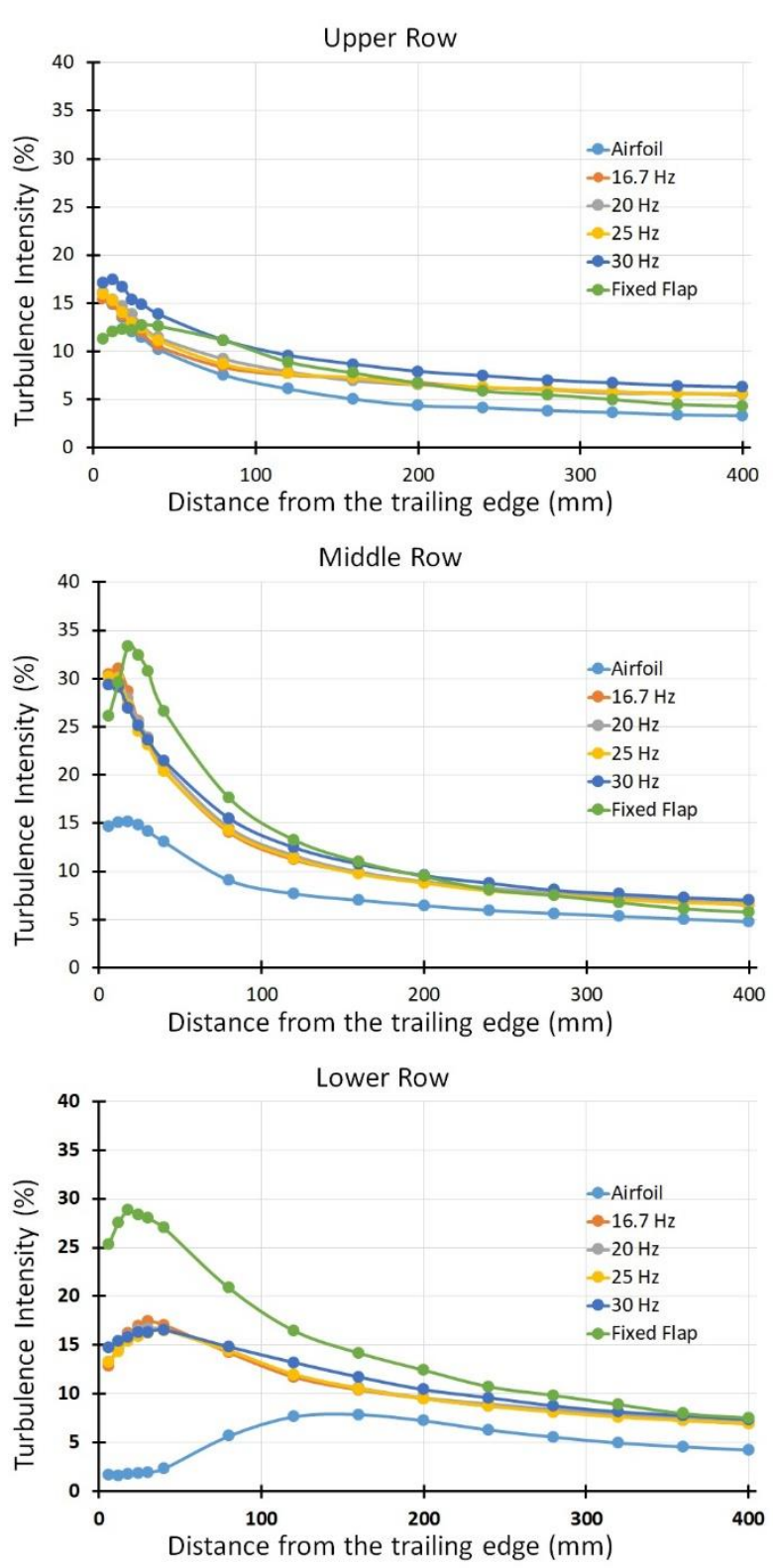

Figure 8. Turbulence Intensity $-\alpha=2^{\circ}$.

both fields and for the different oscillation frequencies, in the following figures.

Watching the autocorrelation function distribution (Fig. 9), it is found that all of them are periodical. In the still GMF case the oscillation frequency produced by the vortex shedding is found. In the same way, for the oscillating GMF cases it is seen that the forcing frequency is imposed. As expected, the shedding frequency is more intense in the near field.

Applying the frozen flow theory, combined with the exponential decay criteria, the temporal and spatial turbulent scales are determined for both velocity components. The values for three configurations, plain airfoil, still GMF and $25 \mathrm{~Hz}$ frequency - the other frequencies show similar results (Figs. 10 and 11).

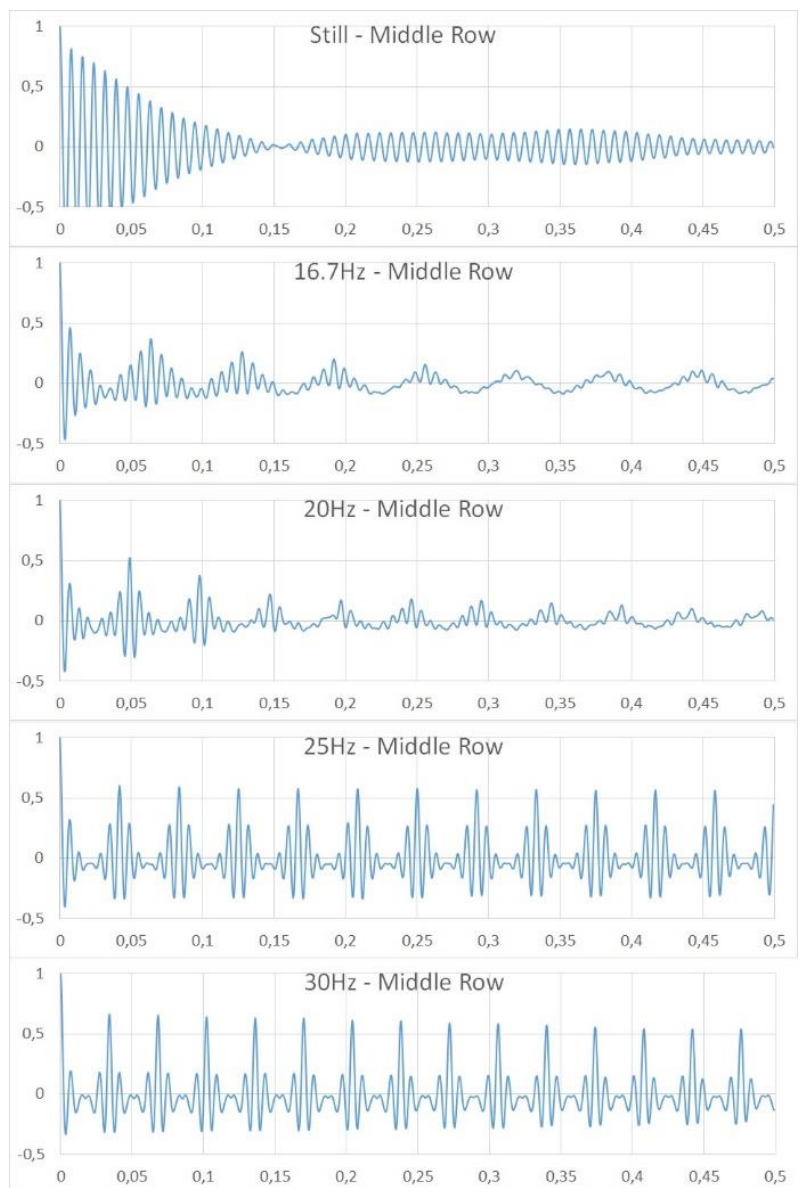

Figure 9. Autocorrelation Distribution Coefficients - Near field.

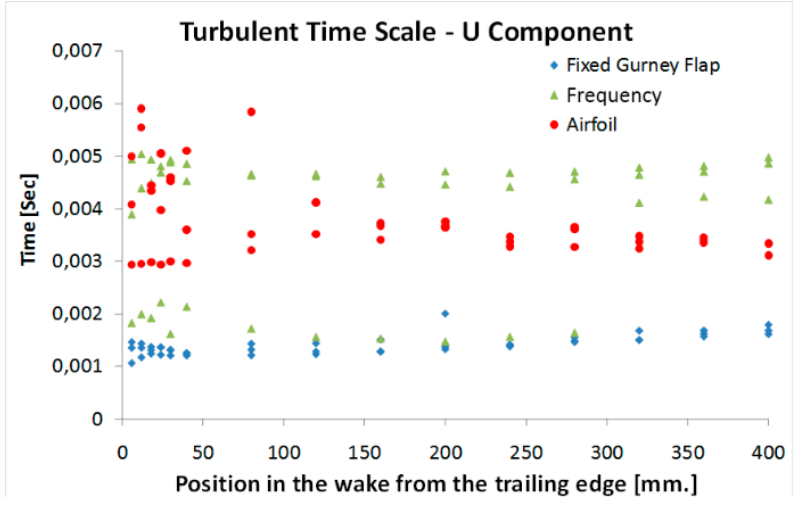

Figure 10. Turbulent time scale $-u$ component.

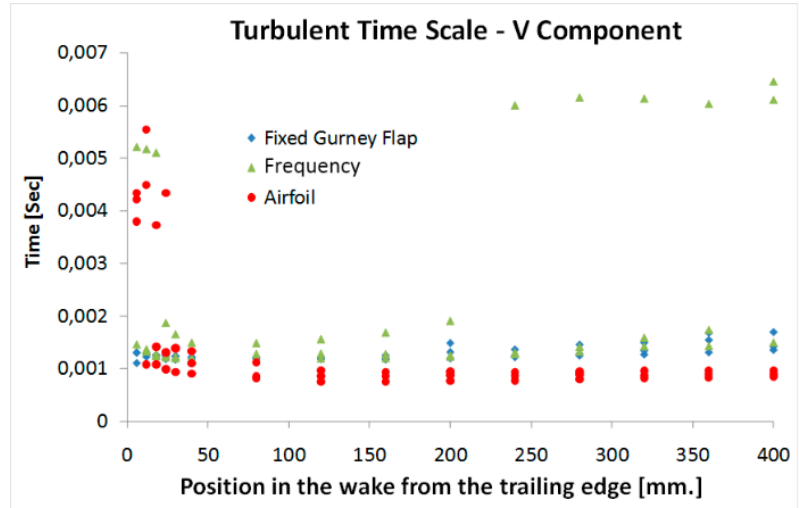

Figure 11. Turbulent time scale $-v$ component. 


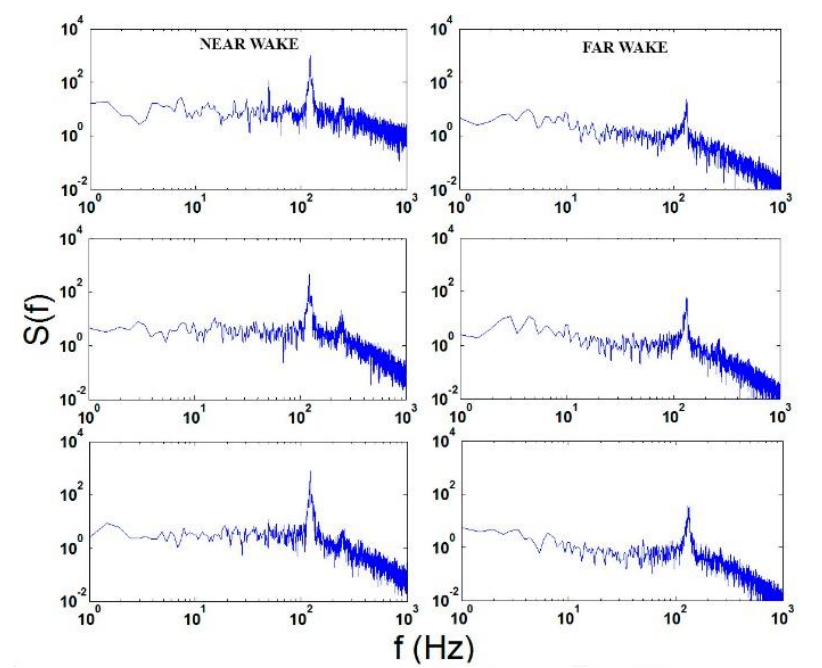

Figure 12. PSD - Fixed Gurney Flap.

In all cases it is seen that the scales are larger at the far wake. In the still GMF configuration and oscillating GMF configurations the scales are very similar. The plain model generates the smallest spatial scales.

Evidence of chaotic flow characteristics is found from both, temporal and spatial scales in the near wake. This is due to the velocities and intensities induced by the vortices generated in that area.

\section{B. PSD Analysis $S(f)$}

Power Spectral Density (PSD) of the signals was determined for the different frequencies and for the different configurations. The two components of the signal were analyzed, plotted (Fig. 12) and tabulated (shown in Tables 1, 2 and 3) to facilitate their study. Results for the three previously mentioned configurations are shown. In all cases, for comparative reasons, the plot representing the near wake $(3 \% \mathrm{C})$ and the plot representing the far wake $(100 \% \mathrm{C})$ are shown side by side; while, each row represents the position of the represented probe (upper, middle and lower).

The PSD peak indicates the relative amount of energy associated with the vortex structures traveling in the wake and their dominant frequency.

From the PSD analysis an increment of power due to the introduction of the GMF - oscillating or still - is observed. Energy peaks are observed in both configurations provided with GMF, while none is observed in the plane airfoil configuration. As expected, in all cases the energy diminishes along the wake as the measure is taken farther from the trailing edge. The detachment of asymmetric, counter-rotating, periodical vortices shed by the still GMF shows such energy decrease along the wake keeping its frequency unaltered. Its effect in the near wake and in the far wake is very strong.

In the oscillating GMF wake, several peaks are detected coinciding the forcing frequency to the largest one. The rest of the peaks correspond to harmonics of the acquired signal. In general, is seen that in the near wake the vortices corresponding to the still GMF are more energetic that those shed by the oscillating GMF. In the far wake the vortices and perturbations generated by the oscillating GMF remains more energetic along the wake than those generated by the still GMF, which decay sooner. The forcing energy introduced with the oscillating GMF seems to be responsible for that effect.

Table 1 to Table 3 show the reference values and energy values in all the field.

\section{DISCUSSION}

The introduction of the still flap in the airfoil is responsible for the increment in lift and drag forces. This is due to the generation of flow instabilities by the shed of counter-rotating, asymmetric vortices (Boldes et al., 2010). It was also proved that the GMF performance changed when submitted to flows with different turbulence characteristics (Colman et al., 2010). The questions arising now are: The efficiency of the airfoil is improved as the wake becomes more turbulent? Is more downwash generated?

The analysis of the instant velocity components for the different configurations, regarding their directions and intensities, analyzed together with the behavior of the model at the different flow conditions, may offer a good approach to the answer. See Fig. 4 to Fig. 6.

From the turbulence intensity, time scales and spatial scales analysis the airfoil wake, with or without the flow control devices, is flow-dynamically characterized. Absolute flow instabilities could be found in laminar and turbulent flow by strong peaks in the PSD charts, as it is shown in Fig. 12, for the fixed Gurney flap vortex shedding.

Values presented in Table 1 validate the hypothesis about nearfield upper and lower vortex strength differ-

Table 1. PSD peaks values for baseline wing section.

\begin{tabular}{|c|c|c|c|c|c|}
\hline & $\mathrm{mm}$. & 6 & 12 & 18 & 24 \\
\hline \multirow{2}{*}{ Upper Row } & $f(\mathrm{~Hz})$ & 155.8 & 183.6 & 162.6 & 50.78 \\
\hline & $S(f)$ & 4.657 & 3.693 & 3.437 & 3.445 \\
\hline Middle & $f(\mathrm{~Hz})$ & 33.69 & 190.4 & 23.44 & 18.55 \\
\hline Row & $S(f)$ & 1.985 & 2.849 & 3.232 & 2.663 \\
\hline \multirow{3}{*}{ Lower Row } & $f(\mathrm{~Hz})$ & 43.46 & 49.8 & 0.9776 & 49.8 \\
\hline & $S(f)$ & 0.1068 & 0.2328 & 0.2367 & 0.2958 \\
\hline & $\mathrm{mm}$. & 30 & 40 & 80 & 120 \\
\hline \multirow[t]{2}{*}{ Upper Row } & $f(\mathrm{~Hz})$ & 6.348 & 117.7 & 191.9 & 151.4 \\
\hline & $S(f)$ & 3.478 & 2.542 & 2.718 & 1.869 \\
\hline Middle & $f(\mathrm{~Hz})$ & 33.2 & 174.8 & 212.4 & 187.5 \\
\hline Row & $S(f)$ & 2.253 & 2.494 & 2.346 & 2.415 \\
\hline \multirow{3}{*}{ Lower Row } & $f(\mathrm{~Hz})$ & 49.8 & 49.8 & 49.8 & 187.5 \\
\hline & $S(f)$ & 0.4644 & 0.8061 & 0.7271 & 0.8511 \\
\hline & $\mathrm{mm}$. & 160 & 200 & 240 & 280 \\
\hline \multirow[t]{2}{*}{ Upper Row } & $f(\mathrm{~Hz})$ & 235.8 & 49.8 & 219.7 & 219.9 \\
\hline & $S(f)$ & 1.942 & 1.728 & 1.492 & 1.784 \\
\hline Middle & $f(\mathrm{~Hz})$ & 247.1 & 175.3 & 206.5 & 223.6 \\
\hline Row & $S(f)$ & 2.417 & 2.198 & 1.979 & 2.064 \\
\hline \multirow{3}{*}{ Lower Row } & $f(\mathrm{~Hz})$ & 247.1 & 243.7 & 233.4 & 243.7 \\
\hline & $S(f)$ & 1.165 & 1.241 & 1.85 & 1.663 \\
\hline & $\mathrm{mm}$. & 320 & 360 & 400 & \\
\hline \multirow[t]{2}{*}{ Upper Row } & $f(\mathrm{~Hz})$ & 238.8 & 211.4 & 49.8 & \\
\hline & $S(f)$ & 1.38 & 1.403 & 1.723 & \\
\hline Middle & $f(\mathrm{~Hz})$ & 228.5 & 211.4 & 247.1 & \\
\hline Row & $S(f)$ & 2.082 & 2.276 & 1.973 & \\
\hline \multirow{2}{*}{ Lower Row } & $f(\mathrm{~Hz})$ & 228.5 & 211.4 & 197.8 & \\
\hline & $S(f)$ & 1,784 & 1.873 & 1.527 & \\
\hline
\end{tabular}


Table 2. PSD values for fixed Gurney flap at $96 \%$ chord length.

\begin{tabular}{cccccc}
\hline & $\mathrm{mm}$. & 6 & 12 & 18 & 24 \\
\hline \multirow{2}{*}{ Upper Row } & $f(\mathrm{~Hz})$ & 24.41 & 23.93 & 23.93 & 23.93 \\
& $S(f)$ & 578.2 & 572.5 & 495.2 & 370 \\
Middle Row & $f(\mathrm{~Hz})$ & 24.41 & 23.93 & 23.93 & 23.93 \\
& $S(f)$ & 91.81 & 184.9 & 224.7 & 211.7 \\
Lower Row & $f(\mathrm{~Hz})$ & 24.41 & 23.93 & 23,93 & 23.93 \\
& $S(f)$ & 213.4 & 143.6 & 79.8 & 56.83 \\
\hline & $\mathrm{mm}$. & 30 & 40 & 80 & 120 \\
\hline Upper Row & $f(\mathrm{~Hz})$ & 23.93 & 23.93 & 23.93 & 23.93 \\
& $S(f)$ & 360.7 & 256 & 146.6 & 168.8 \\
Middle Row & $f(\mathrm{~Hz})$ & 23.93 & 23.93 & 23.93 & 23.93 \\
& $S(f)$ & 234.1 & 266.8 & 148.9 & 138.1 \\
Lower Row & $f(\mathrm{~Hz})$ & 23.93 & 23.93 & 23.93 & 23.93 \\
& $S(f)$ & 61.75 & 96.88 & 118.9 & 82.36 \\
\hline \multirow{4}{*}{ Upper Row } & $\mathrm{mm}$. & 160 & 200 & 240 & 280 \\
& $f(\mathrm{~Hz})$ & 23.93 & 23.93 & 23.93 & 23.93 \\
& $S(f)$ & 150.5 & 17.4 & 141.6 & 128.7 \\
Middle Row & $f(\mathrm{~Hz})$ & 23.93 & 23.93 & 23.93 & 23.93 \\
& $S(f)$ & 113.3 & 91.78 & 113.5 & 108.6 \\
Lower Row & $f(\mathrm{~Hz})$ & 23.93 & 23.93 & 23.93 & 23.93 \\
& $S(f)$ & 49.22 & 33.7 & 29.05 & 28.03 \\
\hline & $\mathrm{mm}$. & 320 & 360 & 400 & \\
\hline Upper Row & $f(\mathrm{~Hz})$ & 23.93 & 23.93 & 23.44 & \\
& $S(f)$ & 120.2 & 125.5 & 108.7 & \\
Middle Row & $f(\mathrm{~Hz})$ & 23.93 & 23.93 & 23.44 & \\
& $S(f)$ & 108 & 119.8 & 107 & \\
Lower Row & $f(\mathrm{~Hz})$ & 23.93 & 23.93 & 23.44 & \\
& $S(f)$ & 29.59 & 22.56 & 24.55 & \\
\hline & & & & &
\end{tabular}

Table 3. PSD values for oscillating Gurney flap at $25 \mathrm{~Hz}$.

\begin{tabular}{cccccc}
\hline & $\mathrm{mm}$. & 6 & 12 & 18 & 24 \\
\hline \multirow{2}{*}{ Upper Row } & $f(\mathrm{~Hz})$ & 120.6 & 122.6 & 127 & 125.5 \\
& $S(f)$ & 620.2 & 977.2 & 820.6 & 787.8 \\
Middle Row & $f(\mathrm{~Hz})$ & 120.6 & 122.6 & 122.6 & 125.5 \\
& $S(f)$ & 71.66 & 484.2 & 838.8 & 1422 \\
Lower Row & $f(\mathrm{~Hz})$ & 120.6 & 122.6 & 126.5 & 125.5 \\
& $S(f)$ & 650.7 & 786 & 463.9 & 663.2 \\
\hline & $\mathrm{mm}$. & 30 & 40 & 80 & 120 \\
\hline Upper Row & $f(\mathrm{~Hz})$ & 126.5 & 128.9 & 129.4 & 131.8 \\
& $S(f)$ & 750.2 & 532.8 & 360 & 171.9 \\
Middle Row & $f(\mathrm{~Hz})$ & 126.5 & 128.9 & 129.4 & 131.8 \\
& $S(f)$ & 1562 & 1240 & 893.3 & 436.3 \\
Lower Row & $f(\mathrm{~Hz})$ & 126.5 & 128.9 & 129.4 & 132.3 \\
& $S(f)$ & 745.8 & 531.8 & 436.7 & 186.9 \\
\hline Upper Row & $\mathrm{mm}$. & 160 & 200 & 240 & 280 \\
& $f(\mathrm{~Hz})$ & 134.8 & 130.9 & 130.9 & 130.9 \\
& $S(f)$ & 160.2 & 81.19 & 65.21 & 37.21 \\
Middle Row & $f(\mathrm{~Hz})$ & 134.8 & 130.9 & 133.8 & 130.9 \\
& $S(f)$ & 391.2 & 186.4 & 158.6 & 110.7 \\
Lower Row & $f(\mathrm{~Hz})$ & 134.8 & 130.9 & 133.8 & 130.4 \\
& $S(f)$ & 213.1 & 103.7 & 80.86 & 57.16 \\
\hline & $\mathrm{mm}$. & 320 & 360 & 400 & \\
\hline Upper Row & $f(\mathrm{~Hz})$ & 134.3 & 133.8 & 132.8 & \\
& $S(f)$ & 49.84 & 25.77 & 23.85 & \\
Middle Row & $f(\mathrm{~Hz})$ & 134.3 & 134.3 & 132.8 & \\
& $S(f)$ & 153.3 & 69.76 & 60.2 & \\
Lower Row & $f(\mathrm{~Hz})$ & 134.3 & 133.8 & 130.9 & \\
& $S(f)$ & 90.4 & 46.12 & 36.21 & \\
\hline & & & & &
\end{tabular}

ences as a mechanism capable of generating extra lift. It is assumed that PSD peaks take place in that zone where vortices start to growth and detach starting their movement into the wake.
The intermittent vortex shedding at Gurney flap upper end have been reported at reference (Troolin et al., 2006), interpreted as a perturbation mechanism acting upon the shear layer coming from the wing section upper surface close to its instantaneous separation point. Therefore the wing section lower surface shear layer separation takes place under different condition compared to that of the baseline wing section, leading to an asymmetrical vortex shedding in the vertical plane.

According to near field wake results presented in this work, it is possible to think that asymmetrical counterrotating vortex generated by the fixed Gurney flap at the trailing edge are the mechanism that lead to lift enhanced. Also, these turbulent flow case results suggest the presence of the same effect reported in laminar flow cases at the same Reynolds number.

\section{CONCLUSIONS}

According to the objective of this work tests were conducted, results were processed and analyzed in order to fluid-dynamically characterize the wake flow and its possible impact on the efficiency of the airfoil provided with an active flow control system.

The wake behind a 2D wing provided by Gurney flap under turbulent flow could be divided into two zones. The near field wake extents from the wing trailing edge up to $10 \%$ wing chord downstream. From that point on, the far field wake takes place.

A periodical detachment of vortices phenomena develops in the near field of the wake, producing an asymmetrical vortex shedding between the upper and the lower trailing edge surfaces which increase the downwash. The wake stabilizes while the spatial turbulent scales increase as it moves beyond 50\% wing chord length. In the oscillating Gurney flap case the energy of the wake does not have a well define behavior, in contrast to the clear energy decay observed in fixed Gurney flap condition.

The appearance of the asymmetric counterrotating vortices in still GMF wake and their frequency of detachment is a consequence of the variation of the pressure in that area and of the movement of the stagnation point. All this generates the characterized turbulent wake. In the case of the oscillating GMF the pressure variation is different, since the movement of the stagnation point is different and depend on the incoming flow, on the geometric characteristics of the GMF and on its relative position on the airfoil. In this case, as shown in Wassen et al. (2017), the vortices generated there present different intensities and scales. This, together with the incoming flow, results in wake with less turbulence intensity than for the still GMF. It would be interesting to make the GMF oscillate at a frequency similar to the detachment frequency since, in this manner, it would be possible to recognize the effect. When the frequency is lower, as in our experiment, several vortices are detached during the GMF movement instead of only one, as would be the ideal situation.

The aim of our work is to generate a more suitable 
induced Gurney flap vortex wake by controlling the evolution time of vortex structures, shedding time rate, vortex strength and scales, etc. as an active flow control mechanism to enhanced aerodynamic performance. In this way we could enhance the application and performance of such Gurney Flap used in racing car spoilers, wind generator blades and aircraft high lift devices at different flight conditions. For example, In racing car could be used in different race conditions enhancing de downforce results. In wind generators blades to change starting torque of the wind turbine in different incident wind conditions

\section{REFERENCES}

Boldes, U., Delnero, J.S., Marañón Di Leo, J., Colman, J. and Camocardi, M. (2010). "The wake asymmetry of an airfoil with a Gurney flap and their connection with the observed lift increase," International Review of Aerospace Engineering, 3, 89- 95.

Casper, M., Scholz, P., Colman, J., Marañón Di Leo, J., Delnero, J. and Camocardi, M. (2012). "Comparison of aerodynamic effects promoted by mechanical and fluidic miniflaps," In: Applied Aerodynamics, Intech Open Access Publishers, 43-62.

Colman, J., Marañón Di Leo, J., Delnero, J.S., Martínez, M., Boldes, U.F. and Bacchi, F. (2010). "Lift and Drag Coefficients Behaviour at Low Reynolds Number in an Airfoil with Miniflap Gurney Submitted to a Turbulent Flow. Part 2," Latin American Applied Research, 40, 75-80.

Delnero, J.S., Marañon Di Leo, J., Bacchi, F., Colman, J. and Boldes. (2005). "Experimental determination of the influence of turbulent scale on the lift and drag coefficients of low Reynolds number airfoils," Latin American Applied Research, 35, 183-188.

Gad-el-Hak, M. (2001). "Flow control: The future," Journal of Aircraft, 38, 402-418.
Giguére, P., Lemay, J. and Dumas, G. (1995). "Gurney Flap Effects and Scaling for Low-Speed Airfoils," 13th AIAA Applied Aerodynamics Conference, AIAA 95-1881, San Diego.

Hah, C. and Lakshminarayana, B. (1982). "Measurement and prediction of mean velocity and turbulence structure in the near wake of an airfoil," Journal of Fluid Mechanics, 115, 251-282.

Jeffrey, D.R.M. and Hurst, D.W. (1996). "Aerodynamics of the Gurney Flap," AIAA Applied Aerodynamic Conference, AIAA 96-2418-CP.

Mahrt, L. (1991). "Eddy asymmetry in the sheared heated boundary layer," J. Atmos. Sci., 48, 472-492.

Schatz, M., Guenther, B. and Thiele, F. (2004). "Computational Modeling of the Unsteady Wake behind Gurney-Flaps," 2nd AIAA Flow Control Conference, AIAA-2417, Portland, Oregon, USA.

Troolin, D.R., Longmire, E.K. and Lai, W.T. (2006). "Time re-solved PIV analysis of flow over a NACA 0015 air-foil with Gurney flap," Experiments in Fluids, 41, 241-254.

Van Dam, C.P., Yen, D.T. and Vijgen, P.M.H.W. (1999). "Gurney Flap Experiments on Airfoil and Wings," Journal of Aircraft, 36, 484-486.

Wassen, E., Günther, B., Thiele, F., Delnero, J.S., Marañon Di Leo, J., Boldes, U., Colman, J., Bacchi, F. and Martínez, M. A. (2007) "A Combined Numerical and Experimental Study of Mini-Flaps at Varying Positions on an Airfoils," 45th AIAA Aerospace Sciences Meeting and Exhibit, Reno, 8-11 January.

Received: July 31, 2018

Sent to Subject Editor: May 5, 2019

Accepted: September 27, 2019

Recommended by Subject Editor M. Luján Ferreira 